\title{
Dificultades de lectura en contextos de pobreza: ¿un caso de Efecto Mateo?
}

\author{
Beatriz Diuk \\ Marina Ferroni
}

\begin{abstract}
Resumen
Este estudio investigó si las dificultades de lectura en contextos de pobreza pueden interpretarse en términos del Efecto Mateo, que plantea una acumulación de diferencias entre quien ingresa a la escuela con altos y bajos conocimientos. Se evaluaron 58 niños recién admitidos en el primer grado con pruebas de procesamiento fonológico, conocimiento de letras, lectura y escritura y se identificó un grupo de niños en riesgo. Con base en evaluaciones de lectura y escritura realizadas al final del primer, segundo y tercer grado se comparó la evolución de los niños en riesgo con la de sus compañeros. En el primer grado, la distancia entre grupos se amplió, confirmando las predicciones del Efecto Mateo. A partir del segundo grado, la escuela proporcionó a los niños en riesgo un apoyo educativo adicional. No se ampliaron más las diferencias entre grupos. Se considera que estos resultados manifiestan la repercusión de la intervención pedagógica en la configuración de las dificultades.
\end{abstract}

Palabras Clave: Lectura, pobreza, dificultades escolares.

\section{Dificuldades de leitura em contextos de pobreza: um caso de Efeito Mateus?}

\section{Resumo}

Este estudo explorou se as dificuldades leitoras em contextos de pobreza podem ser interpretadas em termos do Efeito Mateus, que postula uma acumulação das diferenças entre quem ingressa na escola com altos e baixos conhecimentos. Foram avaliadas 58 crianças ingressantes na primeira série com testes de processamento fonológico, conhecimento de letras, leitura e escrita, e se identificou um grupo de crianças em risco. Com base em avaliações de leitura e escrita ministradas no final da primeira, segunda e terceira série, se comparou a evolução das crianças em risco com a de seus pares. No primeiro ano, a distância entre grupos se ampliou, confirmando as predições do Efeito Mateus. A partir do segundo ano, a escola proporcionou às crianças em risco um apoio educativo adicional. As diferenças entre grupos já não se ampliaram. Considera-se que esses resultados evidenciam a incidência da intervenção pedagógica na configuração das dificuldades.

Palavras-chave: Leitura, pobreza, dificuldades escolares.

\section{Reading difficulties in a poverty context: a case study of the Matthew Effect?}

\begin{abstract}
In this study we investigate whether the reading difficulties in poverty environment may be interpreted in terms of the "Matthew Effect", which posits an accumulation of differences between those who enter school with high and low knowledge. We evaluate 58 children entering first grade with tests of phonological processing, letter knowledge, reading and writing, and identified a group of children at risk. Based on assessments of reading and writing taught at the end of the first, second and third grades, we compared the evolution of children at risk with their peers. In the first year, the gap between groups widened confirming the predictions of the Matthew Effect. From the second year, the school provided children at risk with an additional educational support. Differences between groups no longer increased. It is considered that these results show the impact of educational intervention in facing children's difficulties.
\end{abstract}

Keywords: Reading, poverty, school difficulties. 


\section{Introducción}

El presente trabajo busca contribuir al estudio de la configuración de las dificultades lectoras en niños que crecen en contextos de pobreza. La existencia de una asociación entre nivel socioeconómico y desempeño lector es ampliamente reconocida en la literatura sobre el tema (Andrés, Canet-Juric, Richard's, Introzzi, \& Urquijo, 2010; D’Angiulli, Siegel, \& Hotzman, 2004).

Las explicaciones sobre esta problemática han tendido a polarizarse en torno a dos marcos interpretativos (Espíndola \& León, 2002). Por un lado, se ha propuesto que los niños de los sectores más pobres carecerían de la preparación o de las capacidades necesarias para realizar el aprendizaje lector en los primeros años de escolarización (ver revisión en Lus, 2005). De hecho, la investigación epidemiológica ha mostrado que los niños de ciertos subgrupos socialmente minoritarios tienen muchas mayores posibilidades de ser identificados como niños con déficits de aprendizaje (Klinger, Artiles, \& Barletta, 2006).

Desde una postura alternativa los investigadores de la tradición sociocultural han sugerido que las dificultades que los niños más pobres experimentan resultan del desajuste entre los patrones de uso del lenguaje y los modos de aprendizaje de los niños y sus comunidades y aquellos que la escuela espera y demanda (Borzone \& Rosemberg, 1999, 2000; Dickinson \& McCabe, 2001). Aunque este tipo de interpretación encuentra consenso en ámbitos académicos, el sistema escolar continúa operando en muchos casos en base a la atribución del fracaso en el aprendizaje a las competencias propias de los niños. En este contexto, las investigaciones sobre dificultades lectoras realizadas en el marco de la psicología cognitiva tradicionalmente excluyeron a los niños provenientes de sectores socioeconómicos bajos. Si bien el objetivo de la exclusión fue evitar atribuir a las capacidades de los niños las dificultades que están originadas en inadecuadas oportunidades educativas, en la práctica el resultado fue la desatención de la problemática de los niños en contextos de pobreza (Bravo, Bermeosolo, \& Pinto, 1991).

En el presente estudio se considera que la incorporación de los niños más pobres a los estudios realizados sobre adquisición lectora en el marco de la psicología cognitiva puede contribuir al desarrollo de una perspectiva que permita comprender el modo en que las características constitucionales y el ambiente se articulan para producir las dificultades. El concepto de Efecto Mateo en lectura (Stanovich, 2000) puede resultar particularmente fértil para avanzar en esta comprensión.

El concepto de Efecto Mateo en lectura propone que en ámbitos educativos se produciría un efecto de acumulación de las diferencias, fenómeno por el cual quienes ingresan con mayores conocimientos desarrollan tasas de aprendizaje superiores a las de quienes ingresan con menores habilidades. Las dificultades lectoras se configurarían, entonces, a partir de una intervención pedagógica que no atiende a las necesidades educativas de los niños que ingresan al sistema siendo más vulnerables.
Ahora bien, los intentos por obtener evidencia empírica respecto del efecto Mateo han sido poco exitosos. A pesar de que algunos estudios encontraron una ampliación gradual de la brecha entre buenos y malos lectores (Bast \& Reitsma, 1998) o encontraron la reducción de la brecha en algunos aspectos y su ampliación en otros (Aunola, Leskinen, Onatsu-Arvilommi, \& Nurmi, 2002), la mayor parte reveló un patrón de reducción de la distancia entre grupos (Catts, Hogan, \& Fey, 2003; Parilla, Aunola, Leskinen, Nurmi, \& Kirby, 2005; Scarborough \& Parker, 2003). Dado que estos estudios incorporaron a sujetos provenientes de los sectores medios de la sociedad, es posible pensar que el efecto Mateo difícilmente se produzca entre niños que se encuentran en contextos educativos de alta calidad. Este hecho es atribuible a que, cuando los niños de los sectores medios de la sociedad encuentran dificultades en el proceso de alfabetización, tempranamente comienzan a operar una serie de factores protectores - apoyo familiar, atención por parte de maestros particulares o psicopedagogos - que permitirían reducir la brecha y evitar el desarrollo de dificultades más persistentes (Parilla y cols., 2005).

Pero el efecto Mateo podría dar cuenta de configuración de las dificultades de los niños en contextos de pobreza. En este sentido, resulta notable el estudio de Morgan, Farkas y Hibel (2008), quienes encontraron un Efecto Mateo en lectura asociado a la procedencia étnica y socioeconómica de los niños. Es posible pensar que las familias de los sectores más pobres de la sociedad no cuentan con los recursos para generar por sí solas la red de apoyo que los niños cognitivamente más vulnerables suelen necesitar. Si la escuela no proporciona este apoyo, resulta escasamente sorprendente que un porcentaje de los niños de estos sectores no logre avanzar en su aprendizaje.

El presente estudio se propuso explorar la existencia del Efecto Mateo en niños que asistían a una escuela que atiende a población en contextos de pobreza. Para ello, al comenzar el 1er grado, en base al desempeño en pruebas de reconocimiento de sonido inicial y de conocimiento de letras, que constituyen los mejores predictores de la adquisición lectora (Caravolas, Hulme, \& Snowling, 2001), se identificó a un grupo de niños considerado en riesgo y se comparó durante los tres primeros años de escolarización el aprendizaje de la lectura y la escritura de estos niños con el de sus pares.

Cabe señalar que el estudio que se reporta en este trabajo es producto de una situación particular que tuvo lugar en la escuela de los niños. En efecto, cuando los niños finalizaron el 1er grado, la escuela consideró necesario profundizar la intervención pedagógica destinada a quienes presentaban mayores dificultades. En consecuencia, el presente estudio ha permitido explorar la evolución de los niños más vulnerables durante su primer grado escolar, cuando participaban exclusivamente de la misma intervención pedagógica que sus pares, así como su desempeño a partir del segundo grado escolar, en el que la escuela les proporcionó oportunidades educativas adicionales. En este sentido, se busca responder a las siguientes preguntas: ¿se produce 
una ampliación de la brecha entre los niños más vulnerables y sus pares cuando todos participan de la misma intervención pedagógica en el aula? ¿Es posible enfrentar el efecto Mateo a partir de mejorar las oportunidades educativas de los niños más vulnerables?

Ahora bien, el estudio del aprendizaje de la lectura y la escritura durante los tres primeros años de escolarización requiere de un diseño que contemple la evolución de las habilidades evaluadas. En efecto, se ha propuesto que la ampliación de las brechas encontraría un límite evolutivo en la propia habilidad que se mide: es posible que las diferencias individuales en algún componente se incrementen durante ciertas fases de la adquisición lectora, a partir de lo cual permanecerían estables para dicho componente pero serían identificables en otros componentes, más avanzados, del aprendizaje de la lectura y la escritura (Bast \& Reitsma, 1998).

En consecuencia, la evaluación de los niños se planificó en función de la propuesta que realizara Frith (1985) acerca del solapamiento entre el desarrollo de la lectura y de la escritura, posteriormente validada por distintos autores (Adams, 1990; Ehri, 1992; Share, 1999, 2004). Se considera que el dominio del sistema de escritura se inicia con el aprendizaje de la escritura de palabras a través de la representación de su estructura fonológica, sin atender a la ortografía convencional. Los niños incrementan gradualmente su capacidad de analizar la forma sonora de las palabras y de establecer las correspondencias fonema-grafema, produciendo escrituras que recuperan de modo cada vez más completo el contenido fonológico de las palabras. Logran entonces producir escrituras fonológicamente apropiadas, es decir escrituras en las que cada fonema es representado por un grafema que corresponde a dicho fonema en algún contexto, aunque no necesariamente en la palabra intentada (varco por barco).

El desarrollo de la escritura fonológica constituye en este momento del aprendizaje el elemento dinámico de la adquisición alfabética. La escritura facilita el dominio de las correspondencias fonema-grafema y así promueve el desarrollo de la lectura, que comienza siendo laboriosa y con frecuencia incorrecta, para incrementar gradualmente su precisión. La lectura adquiere entonces un rol de dinamizador del aprendizaje dado que la práctica lectora produce mayor fluidez y facilita la adquisición de conocimiento ortográfico. La lectura fluida y veloz y la escritura de palabras respetando la ortografía convencional constituyen el punto culminante del dominio del sistema de escritura.

En base a este marco, se evaluó a los niños mediante pruebas de lectura y escritura de palabras. En la evaluación del desempeño en estas pruebas se consideró el grado de completamiento fonológico de las escrituras, su corrección ortográfica, la precisión en la lectura así como también la velocidad lectora.

\section{Método}

Sujetos

Participaron de este estudio los niños ingresantes a 1er grado en una escuela parroquial que atiende a niños provenientes de familias en contextos de pobreza de las afueras de Buenos Aires, Argentina. De los 62 ingresantes, 1 niño fue excluido del estudio a pedido de la escuela debido a un trastorno emocional. Del resto del grupo se obtuvo el consentimiento informado de los padres de 58 niños. Dada la alta estabilidad de la matrícula de la escuela, los 58 niños pudieron ser evaluados a lo largo de los tres años que duró el estudio. La edad promedio de los niños al incorporarse al estudio era de 6 años 1 mes (DS: 5 meses). Cabe señalar, sin embargo, que no todos los niños avanzaron en su trayectoria escolar del mismo modo. Como parte de las estrategias diseñadas por la escuela para garantizar a cada niño el tipo de intervención educativa que necesitaba, 5 niños repitieron el 1er grado escolar y 6 niños repitieron el 2 do grado. Todos los niños continuaron participando del estudio independientemente del grado escolar en el que se encontraban dado que el objetivo no era analizar el desempeño de los niños en función del grado sino explorar su aprendizaje lector en el marco de la trayectoria escolar que la escuela estableciera para ellos.

Asimismo, los niños que presentaban mayores dificultades, participaron durante el segundo y tercer año de este estudio de un espacio para promover sus aprendizajes. Esta actividad tenía lugar dentro del horario de la jornada escolar y se desarrollaba en pequeños grupos (hasta 6 niños), a cargo de maestros especialmente contratados para esta tarea. En este espacio se trabajaban las distintas áreas del curriculum. En particular en el caso del proceso de alfabetización las actividades desarrolladas no estaban destinadas a completar el trabajo realizado en clase sino a cerrar la brecha entre el nivel de conocimientos de los niños y las exigencias de su grado escolar. Las docentes seguían los lineamientos de una propuesta (Diuk, 2009) que enfatizaba inicialmente el análisis fonológico de las palabras y su escritura para luego avanzar en el desarrollo de las habilidades lectoras.

\section{Materiales}

El diseño del proyecto contempló algunas pruebas que solamente fueron administradas al comenzar el 1er grado, otras pruebas que fueron aplicadas al comenzar y al finalizar ese mismo año, en tanto las pruebas de lectura y escritura de palabras del Test LEE fueron utilizadas a fines del 1ero, 2do y 3ero.

Pruebas administradas exclusivamente al ingresar a 1er grado

Prueba de apareamiento de sonido inicial: se trata de una prueba adaptada de Signorini y Borzone de Manrique (1996) con tres ítems de ensayo y 10 de prueba. El expe- 
rimentador presenta al niño, con el apoyo de dibujos, una palabra y le pregunta cuál de otras dos palabras comienza como la primera palabra que nombró. Se asigna un punto a cada respuesta correcta. A fin de estimar la confiabilidad por consistencia interna de la prueba se calculó el alpha de Cronbach, que fue de .62.

Conocimiento de letras: se presentaron a los niños 25 letras en imprenta mayúscula y se les pidió que las nombraran. Cada letra fue presentada en forma individual, en Arial, tamaño 150, en el centro de una hoja en blanco. Se aceptaron como respuestas correctas tanto el nombre como el sonido de la letra. Se asignó un punto a cada respuesta correcta. El alfa de Cronbach fue .94.

Pruebas administradas al comenzar y al finalizar el 1er grado escolar

Escritura y lectura de palabras familiares: una misma lista de 8 palabras fue presentada, en primer lugar, para su lectura y luego para la escritura al dictado. La lista estaba formada por tres palabras que los niños suelen aprender a reconocer o a escribir de memoria (mamá, papá, oso), tres palabras con estructura fonológica sencilla (mesa, pelota y caballo) y dos palabras con estructura fonológica compleja (brazo, escuela). Se asignó un punto a cada palabra correctamente leída. Para puntuar la escritura de palabras se utilizó un criterio fonológico: se asignó un punto a cada palabra en la cual todos los fonemas estuvieran representados.

Pruebas administradas al finalizar el primer, segundo y tercer grado

Lectura de palabras Test LEE (Defior Citoler y cols., 2006). Se asignó un punto a cada palabra leída con precisión. En las evaluaciones realizadas a fin del segundo y del tercer grado se computó también el tiempo de lectura.

Escritura de palabras: se seleccionaron 20 palabras del Test LEE (Defior Citoler y cols., 2006) respetando los criterios psicolingüísticos que orientaron la elaboración de la prueba. Se computaron dos puntuaciones: por un lado, se asignó un punto a cada palabra escrita en forma fonológicamente apropiada. Por otra parte, se atribuyó un punto a cada palabra escrita en forma ortográficamente convencional.

\section{Procedimiento}

Los niños fueron evaluados en forma individual en un espacio dentro de su escuela en el mes de abril y nuevamente en el mes de noviembre del primer grado escolar. Los espacios utilizados se encuentran apartados de la zona de aulas y tienen como función permitir la realización de entrevistas entre miembros de la escuela y los padres. El uso de estos espacios fue autorizado para la realización del presente estudio. En cada instancia cada niño participó de dos sesiones en las que se administraron las pruebas en orden fijo. Las pruebas fueron administradas en sesiones individuales, con la excepción de la prueba de escritura que se aplicó en pequeños grupos.
En los años siguientes los niños fueron evaluados en los meses de octubre y noviembre. Las pruebas se administraron en dos sesiones: una sesión individual para la lectura de palabras y una sesión en pequeños grupos para la escritura.

\section{Resultados}

En base a los puntajes obtenidos por los niños en las pruebas de reconocimiento de sonido inicial y de conocimiento de correspondencias se identificó a un subgrupo que ingresó a 1er grado con un bajo nivel de conocimientos prelectores. Se incluyeron en este subgrupo a aquellos niños que hubieran obtenido puntajes correspondientes al 30\% inferior de la muestra en ambas tareas. Quedó así conformado un grupo de niños considerado vulnerable integrado por 17 alumnos (10 niños y 7 niñas) y un grupo de alumnos sin riesgo lector compuesto por 41 sujetos (19 niños y 22 niñas). Los puntajes obtenidos por cada grupo en todas las tareas administradas se presentan en la Tabla 1.

Una primera inspección de los resultados obtenidos por los niños en las pruebas de lectura y escritura a lo largo de los 3 años que duró el estudio mostró que dos alumnos (1 niño y 1 niña) obtuvieron puntajes muy por debajo de la media del grupo (entre 3 y 4 desvíos) en todas las tareas, por lo que fueron identificados como outliers y no fueron incluidos en los análisis subsiguientes. Cabe señalar que al comenzar el 1er grado los niños se encontraban a menos de un desvío de los puntajes obtenidos por el subgrupo en riesgo, esto es, sus puntajes no sugerían que presentaran un nivel de riesgo mayor al del resto del grupo.

Los resultados obtenidos por los subgrupos de niños en riesgo (GR) y sin riesgo (GSR) en las pruebas de lectura y escritura de palabras a lo largo de los tres años se presentan en la tabla 2. La inspección de las distribuciones de los puntajes en cada subgrupo mostró que la prueba de lectura de palabras familiares administrada al comenzar el 1er grado se alejó significativamente de la distribución normal asintótica en el GR. Transformar la variable no mejoró la distribución y el uso de estadísticos no paramétricos no modificó los resultados de los análisis, por lo que se reportan los resultados obtenidos con las variables no transformadas y los estadísticos normales.

Con el objeto de explorar las diferencias entre grupos en los puntajes obtenidos al comenzar y al finalizar el primer grado escolar se realizaron una serie de Anovas de medidas repetidas sobre los puntajes de lectura y escritura de palabras familiares con grupo (GR y GSR) como factor entre sujetos y tiempo (principio y fin de año) como factor intrasujetos. Se encontró tanto un efecto de tiempo $[F(1,54)$ $=112.33, p=0,000]$ para la lectura y $[F(1.54)=53,01, p=$ $0,000]$ para la escritura, como de grupo $[F(1,54)=48.53, p$ $=0,000]$ para la lectura y $[F(1,54)=26.86, p=0,000]$ para la escritura. Es decir, ambos grupos mejoraron entre principio y fin de año y el grupo sin riesgo superó al grupo en riesgo en ambas tareas en las dos ocasiones evaluadas. Por otra parte, se obtuvo una interacción entre el factor tiempo y el factor 
Tabla 1. Puntajes promedio y desvíos estándar obtenidos por ambos grupos en las pruebas administradas.

\begin{tabular}{|c|c|c|c|c|}
\hline \multirow{2}{*}{ Medidas } & \multicolumn{2}{|c|}{ Grupo en riesgo } & \multicolumn{2}{|c|}{ Grupo sin riesgo } \\
\hline & Media & $D S$ & Media & $D S$ \\
\hline Sonido inicial & 6.26 & 1.43 & 8.26 & 1.54 \\
\hline Conocimiento de letras & 9.33 & 3.28 & 19.75 & 4.47 \\
\hline \multicolumn{5}{|c|}{ Escritura palabras familiares } \\
\hline Principio de 1er grado & .40 & 1.05 & 2.70 & 2.31 \\
\hline Fin de 1 er grado & 1.86 & 1.64 & 5.39 & 2.25 \\
\hline \multicolumn{5}{|c|}{ Escritura fonológica de palabras } \\
\hline Fin 1er grado & 2.26 & 2.25 & 11.95 & 5.82 \\
\hline Fin 2do grado & 13.80 & 5.21 & 17.60 & 3.44 \\
\hline Fin 3er grado & 16.60 & 3.50 & 18.73 & 1.78 \\
\hline \multicolumn{5}{|c|}{ Escritura ortográfica de palabras } \\
\hline Fin de 1 er grado & 1.85 & 2.95 & 7.43 & 3.97 \\
\hline Fin 2do grado & 9.20 & 4.64 & 13.00 & 3.41 \\
\hline Fin 3er grado & 10.86 & 2.61 & 13.75 & 2.43 \\
\hline \multicolumn{5}{|l|}{ Lectura palabras familiares } \\
\hline Principio de 1er grado & .53 & .915 & 2.34 & 2.25 \\
\hline Fin de 1 er grado & 2.60 & 1.50 & 6.70 & 1.38 \\
\hline \multicolumn{5}{|l|}{ Lectura de palabras } \\
\hline Fin de 1 er grado & 1.20 & 4.37 & 18.56 & 13.47 \\
\hline Fin 2do grado & 25.86 & 10.92 & 36.92 & 3.54 \\
\hline Fin 3er grado & 36.53 & 3.37 & 38.60 & 3.11 \\
\hline \multicolumn{5}{|l|}{ Tiempo de lectura } \\
\hline Fin 2do grado & 250.80 & 125.45 & 139.48 & 75.74 \\
\hline Fin 3er grado & 111.40 & 35.90 & 89.37 & 42.00 \\
\hline
\end{tabular}

grupo $[F(1,54)=14.351, p=0,000]$ para la lectura y $[F(1,54)$ $=4.555 p=0,000]$ para la escritura, lo que muestra que los grupos no evolucionaron del mismo modo. En efecto, en ambas tareas, el grupo sin riesgo avanzó en su desempeño más que el grupo en riesgo, dando lugar a una ampliación de la brecha entre grupos durante el primer grado escolar.

Durante el 2do y el 3er grado los niños de GR participaron de situaciones de apoyo escolar adicional en pequeños grupos durante el turno tarde. Es por ello que la comparación entre fin de 1 er grado y fin de 3ero es particularmente relevante a los fines de este estudio.

En el caso de la escritura se computó, por un lado, la evolución en la representación del contenido fonológico de las palabras y, por otro, el uso de la forma ortográficamente convencional. Se presentan los resultados por separado. Una serie de Anovas de medidas repetidas llevado a cabo sobre la escritura fonológica con grupo (con y sin riesgo) como factor entre sujetos y grado (1ero y 2do) como factor intrasujetos reveló, entre 1er y 2 do grado, un efecto de grado $[F(1,54)=96.57, p=0,000]$, de grupo $[F(1,54)=39.31, p=$ $0,000]$ y una interacción entre ambos $[F(1,54)=11.27, p=$ $0,001]$ debido a que la distancia entre grupos se redujo en el tiempo considerado. El análisis de la evolución entre fin de 2do y fin de 3ero mostró un efecto de grupo $[F(1,54)=13.76$ , $p=0,000]$ y un efecto de grado $[F(1,54)=11.59 p=0,001]$. En este caso, a diferencia de lo ocurrido entre 1er y 2 do gra- 
do, no hubo interacción entre grado y grupo, revelando que si bien ambos grupos mejoraron su desempeño entre una medición y la siguiente, la distancia entre grupos se mantuvo durante el 3er grado (ver Figura 1).

Figura 1: Evolución del desempeño en escritura de palabras según criterio fonológico entre fin del 1er, 2do y 3er grado escolar por grupo (GR y GSR)

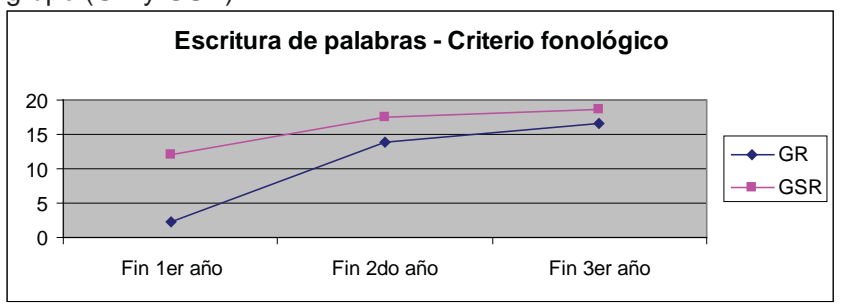

Por su parte, el análisis del dominio de la ortografía convencional presentó un patrón diferente. Tanto entre 1er y 2 do grado como entre 2 do y 3ero se encontraron efectos de grado $[F(1,54)=84.59, p=0,000]$ entre 1ero y 2 do y $[F(1,54)=5.97, p=0,018]$ entre 2 do y 3ero, y de grupo $[F(1,54)=21.34, p=0,000]$ entre 1ero y 2 do y $[F(1,54)$ $=16.37, p=0,000]$ entre 2 do y 3ero, pero en ningún caso se produjo una interacción entre ambos, es decir, la distancia entre grupos se mantuvo.

En el caso de la lectura de palabras, entre fin de 1er grado y fin de 2 do se encontró un efecto de grupo $[F(1,54)=$ 42.16, $p=0,000]$ y de tiempo $[F(1,54)=135.91, p=0,000]$ pero no hubo interacción entre tiempo y grupo $[F(1,54)$ $=3.28, p=0,076]$. Entre fin de 2 do y de 3ero se encontró un efecto de tiempo $[F(1,54)=50.73, p=0,000]$ y de grupo $[F(1,54)=30.03, p=0,000]$ pero en este caso sí se verificó una interacción entre tiempo y grupo $[F(1,54)=24.71, p=$ $0,000]$, asociada al hecho de que el GR incrementó sus puntajes relativamente más que el GSR (ver Figura 2).

Figura 2: Evolución del desempeño en precisión lectora entre fin del 1er, 2do y 3er grado escolar por grupo (GR y GSR)

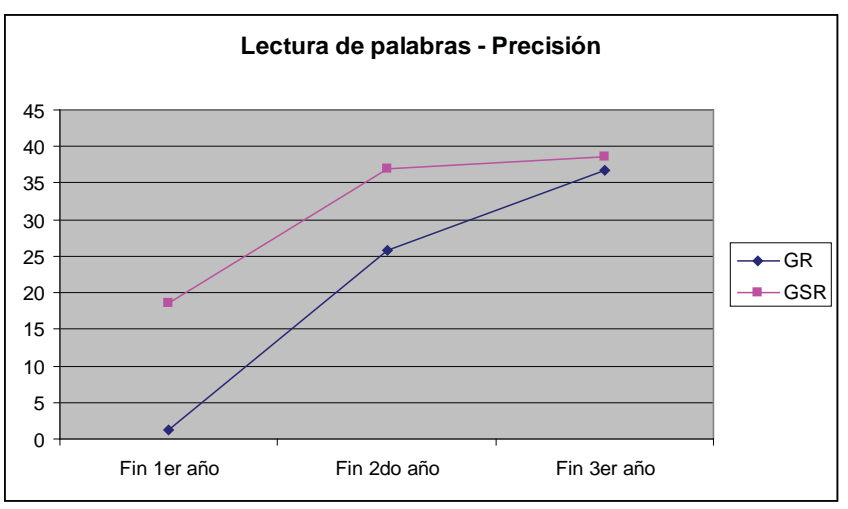

Dado que este resultado podría ser producto de un efecto techo debido a los elevados puntajes iniciales del GSR, se computó también el tiempo de lectura, considerado una medida de la fluidez adquirida por los niños. Se comparó el tiempo lector de cada grupo al finalizar 2 do y 3er grado. Se obtuvo un efecto de tiempo $[F(1,54)=85.20, p=0,000]$ y de grupo $[F(1,54)=21.06, p=0,000]$ y una interacción entre tiempo y grupo $[F(1,54)=22.63, p=0,000]$, debida a que el $\mathrm{GR}$ redujo sus tiempos de lectura en mayor medida que el GSR (ver Figura 3). El conjunto de resultados permite sostener que la diferencia en el desempeño lector entre grupos se había reducido a fin de 3 er grado.

Figura 3: Evolución del tiempo de lectura de palabras entre fin del 2do y 3er grado escolar por grupo (GR y GSR)

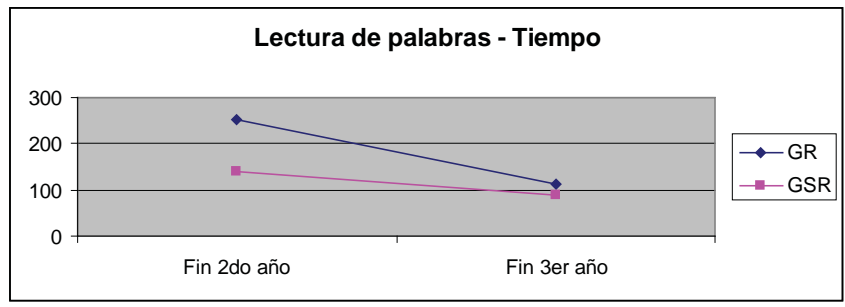

\section{Discusión}

El objetivo del presente estudio fue explorar en qué medida las dificultades lectoras de los niños que crecen en contextos de pobreza pueden interpretarse en términos de la particular interacción entre factores cognitivos y educativos implícita en el concepto de Efecto Mateo. Para ello, un grupo de alumnos ingresantes a 1er grado en una escuela que atiende a niños provenientes de familias de nivel socioeconómico (NSE) bajo fue evaluado mediante una serie de tareas prelectoras y de lectura y escritura de palabras. En base al desempeño en las pruebas de habilidades prelectoras (conocimiento de letras y reconocimiento de sonido inicial), se seleccionó a un grupo de niños considerado en riesgo lector. Se comparó el desempeño de los niños en riesgo con el de sus pares en las pruebas de lectura y escritura de palabras administradas al comenzar y al finalizar el año. Se encontró que la distancia en los puntajes se amplió durante el primer grado escolar. Tal como postula el Efecto Mateo (Stanovich, 1986, 2000), quienes ingresaron a la escuela con un mejor nivel de conocimientos y habilidades prelectoras avanzaron más que sus pares menos aventajados. Este resultado sugiere que, al menos en parte, las dificultades lectoras de los niños que crecen en contextos de pobreza se configuran al interior del sistema educativo.

Ahora bien, a lo largo de los siguientes dos años la brecha entre grupos dejó de ampliarse y en las sucesivas evaluaciones permaneció constante o se redujo. Resulta interesante analizar este patrón evolutivo en el marco del 
modelo propuesto por Frith (1985), en el que sucesivamente distintos componentes del proceso de lectura y escritura se constituyen en el factor dinámico del aprendizaje. En coincidencia con el planteo de esta autora, se encontró que durante el 2do grado escolar (esto es, entre las mediciones de fin de 1 ero y fin de 2do) se redujo la brecha entre grupos en la prueba de escritura fonológica pero no en la escritura ortográfica ni en la lectura de palabras. Es decir, los niños del grupo en riesgo aceleraron su dominio de las fases iniciales de la adquisición del sistema de escritura aunque no lo suficiente como para impactar sobre las habilidades más avanzadas, logro que recién tuvo lugar un año más tarde. Es posible pensar que, si bien el ritmo de aprendizaje de los niños se aceleró como para comenzar a cerrar la brecha con sus compañeros en algún aspecto del proceso, no fue suficiente para una equiparación en tan sólo un año.

En efecto, propone Frith (1985) que el relativo dominio de los mecanismos de escritura fonológica impacta sobre las habilidades de recodificación fonológica en la lectura, que se constituyen a su vez en el factor dinámico en la adquisición de conocimiento ortográfico, conocimiento que se manifiesta en la mayor velocidad lectora y luego en la escritura ortográfica. En el caso del presente estudio los resultados obtenidos responden a las predicciones del modelo. La evolución en la escritura fonológica verificada durante el 2do grado dio lugar, durante el 3er grado, a una reducción de la brecha entre grupos en la precisión y la velocidad lectoras, aunque sin llegar a impactar aún en el dominio de la ortografía convencional al escribir.

Estos resultados contrastan con los obtenidos por Aunola y cols. (2002), quienes encontraron que la reducción en la precisión lectora, debido a un efecto techo, se acompañaba de una ampliación de la brecha en fluidez de la lectura. Es, decir, no se producía una verdadera reducción de las distancias sino que se trasladaban de un componente lector a otro. En este estudio la reducción de las distancias en un componente no se acompañaba de su ampliación en otro, sugiriendo que efectivamente el desempeño del grupo en riesgo comenzaba a aproximarse al de sus pares.

Cabe destacar, sin embargo, que dos niños entre los 58 incorporados al estudio no lograron realizar avances significativos en su aprendizaje de la lectura y la escritura. Se trata de niños que no fueron identificados, al ingresar al estudio, como particularmente vulnerables desde el punto de vista de las habilidades evaluadas. En consecuencia, cabe pensar que los factores de riesgo, en estos niños, residían en aspectos no incorporados al estudio, tales como factores emocionales. Futuras investigaciones deberían incluir estas variables en sus análisis.

El conjunto de resultados obtenidos muestra un claro Efecto Mateo durante el primer grado escolar, pero la desaparición de este efecto en segundo y tercero. Si bien el presente no es un estudio en el cual la variable pedagógica haya sido controlada de modo experimental, creemos que es posible hipotetizar que el hecho de que los niños con mayores dificultades iniciales dejaron de distanciarse de sus compañeros a partir del segundo año se relacionó con las estrategias desplegadas por la escuela, en particular con haber recibido un apoyo educativo adicional. En este sentido el estudio coincide, por un lado, con las investigaciones que identificaron un Efecto Mateo entre los niños más pobres (Morgan y cols., 2008) pero dan apoyo a la interpretación que propusimos en la introducción, en el sentido de que cuando se genera una red de contención el Efecto Mateo desaparece (cf. Parrila y cols., 2005).

En efecto, si bien tal como se señaló, no es este un estudio experimental que permita sostener una relación causal entre intervención pedagógica y aprendizaje, nos inclinamos a pensar que los resultados obtenidos serían el producto de las estrategias implementadas por la escuela fundamentalmente la creación de un espacio de apoyo en pequeños grupos durante el turno tarde - que resultaron exitosas para incrementar el desempeño de los niños vulnerables. Esta afirmación se apoya en el hecho de que la intervención pedagógica implementada tenía como ejes el desarrollo de la lectura y la escritura a partir de incrementar las habilidades de procesamiento fonológico en los niños, estrategia que ha demostrado ser productiva en niños de diversos sectores sociales (Bus \& van ljzendoorn, 1999; Capovilla, A. G. S. \& Capovilla, F. C., 2000; Ehri y cols., 2001; Pestun, Omote, Barreto, \& Matsuo, 2010)

En este sentido, el estudio tiene relevantes implicancias educacionales. Por un lado, cuestiona las interpretaciones tradicionales que atribuyen las dificultades lectoras a las capacidades de los niños. Los resultados de este estudio muestran que las capacidades de los niños interactúan con estrategias de enseñanza que pueden promover u obstaculizar el aprendizaje. Como señalan Baquero, Cimolai, Pérez y Toscano (2005), las posibilidades de aprendizaje no pueden ser evaluadas como una condición a priori sino que sólo pueden ser comprendidas como el efecto de la interacción entre las características del sujeto y las situaciones en las que están implicados.

Las diferencias iniciales entre los niños no pueden interpretarse como capacidades que algunos tendrían y otros no sino como indicadores de experiencias previas cuyo impacto en el aprendizaje posterior depende críticamente de las situaciones educativas que se ofrezcan a los niños. La investigación cognitiva ha mostrado que en tanto quienes ingresan a la escuela con mayor desarrollo prelector aprenden a leer y a escribir sin dificultades en todos los contextos sociales y educativos, los niños cognitivamente más vulnerables dependen de las oportunidades que proporcione el entorno, particularmente en términos de adecuación de las estrategias de enseñanza (Noble, Farah, \& McCandliss, 2006; Ziegler \& Goswami, 2006). Así, el conjunto de resultados obtenido da apoyo al supuesto básico del concepto de Efecto Mateo, esto es, a la idea de que las dificultades lectoras se configuran en la interacción entre cierta vulnerabilidad presente en algunos niños y el modo como la escuela procesa estas características. El estudio sugiere que la vulnerabilidad inicial solamente se convertirá en una dificultad si no se proporciona a los niños las oportunidades educativas adecuadas. 
En este sentido, sería sumamente interesante el desarrollo de futuros proyectos de investigación en los cuales se evalúe el nivel de respuesta de los niños más vulnerables a intervenciones pedagógicas controladas. Se considera en la actualidad que si las dificultades de un niño se relacionan con la falta de oportunidades educativas, presentará, ante una intervención pedagógica adecuada, un alto nivel de respuesta a la intervención (Mceaneaney, Lose, \& Schwartz, 2006; Vaughn \& Fuchs, 2003 ). Estudios de esta naturaleza aportarían una valiosa evidencia acerca de la importancia de la enseñanza en la configuración o la prevención de las dificultades que experimentan los niños que crecen en contextos de pobreza.

\section{Referencias}

Adams, M. (1990). Beginning to read. Thinking and learning about print. Cambridge: The MIT Press.

Andrés, M. A., Canet-Juric, L., Richard's, M., Introzzi, I., \& Urquijo, S. (2010). Disponibilidad de recursos materiales en el hogar y adquisición de habilidades pre-lectoras. Psicología Escolar e Educacional, 14(1), 169 - 148.

Aunola, K., Leskinen, E., Onatsu-Arvilommi, T., \& Nurmi, J. E. (2002). Three methods for studying developmental change: A case of reading skills and self-concept. British Journal of Educational Psychology, 72, 343-364.

Baquero, R., Cimolai, S., Pérez, A., \& Toscano, A. (2005). Las prácticas psicoeducativas y el problema de la educabilidad: La escuela como superficie de emergencia. Revista IIPSI, 8, 121137.

Bast, J., \& Reitsma, P. (1998). Analyzing the development of individual differences in terms of Matthew effects in reading: Results from a Dutch longitudinal study. Developmental Psychology, 34, 13731399.

Borzone de Manrique, A. M., \& Rosemberg, C. (1999). El fracaso escolar: el caso de las comunidades collas. Revista Argentina de Educación, 26, 29-46.

Borzone de Manrique, A. M., \& Rosemberg, C. (2000). Aprender a leer y a escribir entre dos culturas. Buenos Aires: Aique.

Bravo, L., Bermeosolo, J., \& Pinto, A. (1994). El efecto lectura inicial y rendimiento escolar básico. Boletín de Investigación Educacional, 2, C.P.E.I.P. MINEDUC.

Bus, A. G., \& van ljzendoorn, M. H. (1999). Phonological awareness and early reading. A metanálisis of experimental training programs. Journal of Educational Psychology, 91, 403-414.

Capovilla, A. G. S., \& Capovilla, F. C. (2000). Efeitos do treino de consciência fonológica em crianças com baixo nível sócioeconômico. Psicologia Reflexão Crítica, 13(1), 07-24.
Caravolas, M., Hulme, C., \& Snowling, M. J. (2001). The foundations of spelling ability: Evidence from a 3-year longitudinal study. Journal of Memory \& Language, 45, 751-774.

Catts, H., Hogan, T. P., \& Fey, M. (2003). Subgrouping poor readers on the basis of reading-related abilities. Journal of Learning Disabilities, 36, 151-164.

D’Angiulli, D., Siegel, L. S., \& Maggi, S. (2004). Literacy instruction, SES, and word-reading achievement in English-language learners and children with English as a first language: a longitudinal study. Learning Disabilities Research \& Practice, 19, 202-213.

Defior Citoler, S., Fonseca, L., Gottheil, B., Aldrey, A., Jimenez Fernandez, G., Pujals, M., Rosa, G., \& Serrano Chica, F.D. (2006). Test de lectura y escritura en español. Buenos Aires: Paidós.

Dickinson, D. K., \& Mc Cabe, A. (2001). Bringing it all together: The multiple origins, skills and environmental supports of early literacy. Learning Disabilities Research and Practice,16(4), 186-202.

Diuk, B. (2009). Propuesta DALE! Guía para el docente. Buenos Aires: Etis, Save the Children, AECID.

Ehri, L. C. (1992). Reconceptualizing the development of sight word reading and its relationship to recoding. En P. B. Gough, L. C. Ehri y R. Treiman (Eds.), Reading acquisition (pp.107-143). Hillsdale, $\mathrm{NJ}$ : Erlbaum.

Ehri, L. C., Nunes, S. R., Willows, D. M., Schuster, D. V., YaghoubZadeh, Z., \& Shanahan, T. (2001). Phonemic awareness instruction helps children learn to read: Evidence from the National Reading Panel's meta-analysis. Reading Research Quarterly, 36, 250-287.

Espíndola, E., \& León, A. (2002). La deserción escolar en América Latina: un tema prioritario para la agenda regional. Revista Iberoamericana de Educación, 30, 39-62.

Frith, U. (1985). Beneath the surface of developmental dyslexia. En K. E. Patterson, J. C. Marshall \& M. Coltheart (Eds.), Surface dyslexia: Neuropsychological and cognitive studies of phonological reading. (pp. 67-84). London: Erlbaum.

Klinger, J. K., Artiles, A. J., \& Barletta, L. M. (2006). English language learners who straggle with reading: Language acquisition or LD? Journal of Learning Disabilities, 39, 108-128.

Lus, M. A. (2005). De la integración escolar a la Escuela integradora. Buenos Aires: Paidós.

Mceaneaney , J., Lose, M. K., \& Schwartz, R. (2006). A transactional perspective on reading difficulties and Response to Intervention. Reading Research Quarterly, 41, 117- 128.

Morgan, P. L., Farkas, G., \& Hibel, J. (2008). Matthew Effects for Whom? Learning Disabilities Quarterly, 3, 1-12. 
Noble, K. G., Farah, M. J., \& McCandliss, B. D. (2006). Socioeconomic background modulates cognition-achievement relationships in reading. Cognitive Development, 21, 349-368.

Parrila, R., Aunola, K., Leskinen, E., Nurmi, J.-E., \& Kirby, J. R. (2005). Development of individual differences in reading: Results from longitudinal studies in English and Finnish. Journal of Educational Psychology, 97, 299-319.

Pestun, M. S., Omote, L. C., Barreto, D. C., \& Matsuo, T. (2010). Estimulação da consciência fonológica na educação infantil: prevenção de dificuldades na escrita. Psicol. Esc. Educ., 14, 1- 95.

Scarborough, H. S., \& Parker, J. L. (2003). Matthew effects in children with learning disabilities: Development of reading, IQ, and psychosocial problems from grade 2 to grade 8 . Annals of Dyslexia, 53, 47-71.

Share, D.(1999). Phonological recoding and orthographic learning: a direct test of the self-teaching hypothesis. Journal of Experimental Child Psychology, 72, 95-129.

Share, D. (2004). Orthographic learning at a glance. Journal of Experimental Child Psychology, 87, 267-289.
Signorini, A., \& Borzone, A. M. (1996) Las habilidades metafonológicas, la lectura y la escritura en niños de cinco años. Lenguas Modernas, 23, 71-93.

Stanovich, K. E.(1986). Cognitive processes and the reading problems of learning disabled children: Evaluating the assumption of specificity. In J. Torgesen \& B. Wong (Eds.), Psychological and educational perspectives on learning disabilities (pp. 87-131). New York: Academic Press.

Stanovich, K. E. (2000). Progress in understanding reading reading: Scientific foundations and new frontiers. Nueva York-Londres: The Guilford Press.

Vaugh, S., \& Fuchs, L.S. (2003). Redefining learning disabilities as inadequate response to instruction: The promise and potential problems. Learning Disabilities Research and Practice, 18, 137146.

Ziegler, J., \& Goswami, U. (2006). Becoming literate in different languages: similar problems, different solutions. Developmental Science, 9(5), 429-436.

\section{Acerca de los autores}

\section{Beatriz Diuk}

Miembro de la Carrera del Investigador Científico del Consejo Nacional de Investigaciones Científicas y Técnicas de Argentina (CONICET). Doctora en Psicología.

\section{Marina Ferroni}

Becaria de doctorado del Consejo Nacional de Investigaciones Científicas y Técnicas (CONICET). Licenciada en Letras.

\section{Reconocimiento de apoyo financiero}

Esta investigación ha sido financiada por el Consejo Nacional de Investigaciones Científicas y Técnicas (PICT 1388/06) y la Universidad Nacional de San Martín. 